\title{
Epidemiology of early esophageal adenocarcinoma
}

\author{
Thuy-Van P. Hang ${ }^{1}$, Zachary Spiritos ${ }^{2}$, Anthony M. Gamboa ${ }^{3}$, Zhengjia Chen ${ }^{4}$, Seth Force ${ }^{5}$, Vaishali Patel', Saurabh Chawla', \\ Steven Keilin ${ }^{1}$, Nabil F. Saba ${ }^{6}$, Bassel El-Rayes ${ }^{6}$, Qiang Cai ${ }^{1}$, Field F. Willingham ${ }^{1}$
}

${ }^{1}$ Department of Medicine, Division of Digestive Diseases, Emory University, Atlanta, GA; ${ }^{2}$ Department of Medicine, Division of Gastroenterology, Duke University, Durham, NC; ${ }^{2}$ Department of Medicine, Division of Gastroenterology, Hepatology, and Nutrition, Vanderbilt University, Nashville, TN; ${ }^{4}$ Department of Biostatistics and Bioinformatics, Rollins School of Public Health, Emory University, Atlanta, GA; ${ }^{5}$ Department of Surgery, Division of Cardiothoracic Surgery, Emory University, Atlanta, GA; ${ }^{6}$ Emory Winship Cancer Institute, Department of Hematology and Medical Oncology, Emory University, Atlanta, GA, USA

Background/Aims: Endoscopic resection has become the preferred treatment approach for select early esophageal adenocarcinoma (EAC); however, the epidemiology of early stage disease has not been well defined.

Methods: Surveillance Epidemiology and End Results (SEER) data were analyzed to determine age-adjusted incidence rates among major epithelial carcinomas, including EAC, from 1973 to 2017. The percent change in incidence over time was compared according to tumor subtype. Early T-stage, node-negative EAC without metastasis was examined from 2004 to 2017 when precise T-stage data were available.

Results: The percent change in annual incidence from 1973 to 2017 was $767 \%$ for EAC. Joinpoint analysis showed that the average annual percent change in EAC from 1973 to 2017 was 5.11\% (95\% confidence interval, 4.66\%-5.56\%). The annual percent change appeared to plateau between 2004 and 2017; however, early EAC decreased from 2010 to 2017, with an annual percent change of -5.78\%. Conclusions: There has been a 7-fold increase in the incidence of EAC, which was significantly greater than that of the other major epithelial malignancies examined. More recently, the incidence of early EAC has been decreasing. Approximately one in five patients has node negative, potentially resectable early stage disease.

Keywords: Adenocarcinoma; Barrett’s esophagus; Epidemiology; Esophageal neoplasm

\section{INTRODUCTION}

The American Cancer Society estimates that there will be 19,260 new cases and 15,530 deaths from esophageal cancer in 2021. ${ }^{1}$ Among men, esophageal cancer is the seventh leading

\footnotetext{
Received: May 7, $2021 \quad$ Revised: September 27, 2021

Accepted: October 11, 2021

Correspondence: Field F. Willingham

Department of Medicine, Division of Digestive Diseases, Emory University School of Medicine, 1365 Clifton Road, NE, Building B - Suite 1200, Atlanta, GA 30322, USA

E-mail: field.willingham@emory.edu
}

(a) This is an Open Access article distributed under the terms of the Creative Commons Attribution Non-Commercial License (http://creativecommons.org/licenses/by-nc/4.0/) which permits unrestricted non-commercial use, distribution, and reproduction in any medium, provided the original work is properly cited. cause of cancer-related death in the United States (US), with a lifetime risk of one in $125 .{ }^{1}$ Previous studies demonstrated the increasing incidence of esophageal adenocarcinoma (EAC) through the 1990s, ${ }^{2}$ with a transition around 1996 when the adenocarcinoma subtype surpassed squamous cell carcinoma as the most incident esophageal cancer. ${ }^{3}$ While incidence rates have increased, survival for localized EAC has improved with evolving medical, surgical, and endoscopic therapies. ${ }^{4}$ The median survival of patients with localized EAC has increased from 11 to 35 months from the 1970 s to the 2010s. The evolution of endoscopic therapies with good outcomes for early stage disease has led to revisions in national guidelines, which now recommend endoscopic resection followed by radiofrequency ablation for intramucosal and T1a cancers. ${ }^{6}$ However, the epidemiology of this early, potentially locally resectable disease has 
not been examined. This study evaluated the overall incidence and rate of change of EAC relative to other major epithelial cancers, as well as the incidence of early EAC.

\section{METHODS}

\section{Overview}

The National Cancer Institute's Surveillance, Epidemiology, and End Results (SEER) Program is a comprehensive population-based database on cancer incidence and survival in the US, covering approximately $35 \%$ of the US population. ${ }^{7}$ The data are publicly available and de-identified for scientific research.

\section{Data abstraction}

SEER 9 incidence rates for EAC, esophageal squamous cell carcinoma, melanoma, liver and intrahepatic bile duct, kidney and renal pelvis, prostate, breast, bladder, pancreatic, lung and bronchus, colon and rectum, stomach, and cervical cancers from 1973 to 2017 were obtained using rate sessions in the SEER ${ }^{\star}$ Stat ver. 8.3.8. ${ }^{7}$ Separate sessions were performed for each epithelial cancer, as defined by the International Classification of Diseases for Oncology (ICO-3)/World Health Organization (WHO) 2008 anatomic locations, to determine the incidence rates per 100,000 individuals that were age-adjusted to the 2000 US population. The incidence rates of EAC according to race, sex, and age were also examined. In the SEER database, EAC tumor, node and metastasis cancer staging was not documented prior to 2004; therefore, data from 2004 onwards were used to examine early EAC incidence.

\section{Tumor classification}

Tumor staging was performed according to the American Joint Committee on Cancer (AJCC) staging. SEER data from 2004 to 2015 utilized the sixth edition for staging, in which stage 0 tumors are Tis tumors (carcinoma in situ) that are lymph node negative (N0) and have no evidence of metastasis (M0). ${ }^{8}$ Stage I involves T1 N0 M0 tumors, which invade the lamina propria or submucosa but have no lymph node or distant metastases. ${ }^{8}$ Presently, T1 is further subclassified as T1a (invading the lamina propria or muscularis mucosa) and $\mathrm{T} 1 \mathrm{~b}$ (invading the submucosa). These newer subclassifications are available in the SEER database from 2010 onwards under the seventh edition staging. ${ }^{9}$ The analysis of the stages, including T1a and T1b data, was performed on data from 2010-2017 using the seventh edition staging classifications. In this sys- tem, stage IA tumors invade the lamina propria or muscularis mucosa, while stage IB tumors penetrate into the submucosa.' Early EAC is defined as early T-stage tumors (stage 0 and stage I [Ia and Ib]), without nodal spread or distant metastases.

\section{Statistical analysis}

The US National Cancer Institute's Joinpoint Regression Program ver. 4.8.0.1, ${ }^{10}$ was used to analyze trends in age-adjusted cancer incidence rates for epithelial malignancies from 1973 to 2017. This program constructed best-fitting linear regression lines connected via Joinpoints. A maximum of five Joinpoints were generated for each cancer based on the number of intervals with significant trend changes.

For each segment, the annual percentage rate change (APC) and $95 \%$ confidence intervals were reported. APC was defined as:

$$
\left(e^{b}-1\right) \times 100
$$

where $\mathrm{b}$ denotes the slope of the linear line. The weighted average of the APCs was used to calculate the average APC (AAPC), in which the weight was equal to the length of each linear line during the interval. Statistical significance was set at $p<0.05$ for all tests.

The change in the incidence rate for each epithelial malignancy relative to its historic incidence rate in 1973 was represented by the following formula:

$$
100 \times[(\text { rate at a specific year }-1973 \text { rate }) / 1973 \text { rate }]
$$

The change in the incidence of early EAC over time was also examined using Joinpoint analysis from 2010 onwards. To determine the change in the incidence of early stage EAC over time, a rate session was run using the Derived AJCC Stage Group (sixth edition) to obtain the annual incidence of early stage (stage 0-I) versus later stage (stage II-IV) EAC from 2004 to 2015. To determine the overall proportion of early EAC cases, a rate session was run on cases from 2010 to 2017 using Derived AJCC T, N, and M classifications in order to study T1a and T1b subgroups, which were only available with seventh edition staging.

\section{Ethical statements}

The study was reviewed and found to be exempt from review by the Emory University School of Medicine Institutional Review Board. 


\section{RESULTS}

\section{Patient characteristics}

Using the SEER 9 incidence database, 19,619 cases of EAC from 1973 to 2017 were identified. Those with EAC were predominantly men, comprising $85.48 \%$ of the patients. At the time of diagnosis, $7.38 \%$ were less than 50 years old, $18.71 \%$ were 50-59 years old, $30.42 \%$ were $60-69$ years old, $27.07 \%$ were $70-79$ years old, and $16.43 \%$ were aged 80 or older. Regarding racial demographics, $95.32 \%$ were white, $2.11 \%$ black, and 2.57\% American Indian, Alaskan Native, Asian, or Pacific Islander.

\section{Incidence of EAC compared to other epithelial malignan- cies}

Based on the Joinpoint analysis, the incidence rate of EAC significantly outperformed the other major epithelial malignancies (Table 1). In contrast, esophageal squamous cell carcinoma had the second greatest rate of decrease in incidence with an AAPC of $-2.13 \%$. (95\% confidence interval [CI], $-2.53 \%$ to $-1.74 \%$ ). The average annual percent change in EAC from 1973 to 2017 was $5.11 \%$ (95\% CI, 4.66\%-5.56\%). The annual percent change in EAC incidence was statistically significant from 1973 to 1992 and from 1992 to 2004, with APCs of 9.16\% and 4.15\%, respectively. However, from 2004 to 2017, the APC of EAC incidence was insignificant at $0.32 \%$.

The next most rapidly increasing malignancy examined was melanoma of the skin, which had an AAPC of 3.07\% (95\% CI, $2.75 \%-3.39 \%$ ) While the incidence rates of liver and intrahepatic bile duct, kidney and renal pelvis, and breast cancers have increased significantly over time, the incidence rates of colorectal, gastric, esophageal squamous cell carcinoma, and cervical cancer have decreased significantly.

In 1973, there were 0.3 cases of EAC per 100,000 individuals, compared to 2.6 in 2017. The percent change in the annual incidence of EAC was $766.67 \%$ higher in 2017 compared to 1973 (Fig. 1). Esophageal squamous cell carcinoma decreased by $60.71 \%$ over the same time period (Fig. 1). Among the other epithelial malignancies analyzed, melanoma had the second highest rate of increase in annual incidence over the time period (273.53\%) (Fig. 1).

\section{EAC incidence by race and sex}

The incidence rate of EAC has increased over time in all three racial groups identified in the SEER database: white, black, and other (American Indian, Alaskan Native, Asian, Pacific Islander). As shown in Figure 2, the increasing incidence rate of EAC is primarily driven by cases in white men. EAC in white men has increased by more than 9-fold, from 0.64 in 1973 to 6.04 in 2017.

\section{Incidence and proportion of early stage EAC}

T-stage data became available in 2004 in the SEER dataset, with AJCC 6th edition staging applied from 2004 to 2015 and the AJCC 7th edition staging used from 2010 onwards. Among cases of EAC from 2004 to 2015, the incidence of early stage cancers initially appears to be stably elevated at approximately 0.45 per 100,000 cases (Fig. 3 ).

More precise T-stage data (T1a and T1b) became available starting with the 2010 dataset. In the 2010 to 2017 cohort, 19\% of EAC were considered to be at an early stage, of which $1 \%$ were Tis N0 M0, $10 \%$ were T1a N0 M0, $4 \%$ were T1b N0 M0, and $4 \%$ were T1 NOS N0 M0 (Fig. 4). Eighteen percent were stage II EAC at the time of diagnosis, 25\% were stage III, and $38 \%$ were stage IV during the same 7-year period (Fig. 4).

Based on the Joinpoint analysis, the incidence of early EAC (Stage 0, IA, and IB) appeared to decrease from 2010 to 2017 with an AAPC of $-5.78 \%$ ( $95 \% \mathrm{CI},-10.13 \%$ to $-1.21 \%$ ), as can be seen in Figure 5.

\section{DISCUSSION}

While progress has been made in the prevention of several major epithelial cancers over the past three decades, ${ }^{11-13}$ the incidence of EAC has increased significantly. We found that the incidence of EAC has increased at an average rate of $5.11 \%$ per year, with an overall $767 \%$ change in the annual incidence relative to 1973. The rise in EAC compared to squamous cell carcinoma appears to be a true increase in disease burden rather than a result of reclassification of cancers or over-diagnosis due to increased use of diagnostic upper endoscopy methods. ${ }^{3}$ Relative to the other epithelial malignancies, EAC has shown the greatest rate of increase. The increase in the overall rate of EAC agrees with other analyses of total disease burden. ${ }^{4,14-16}$ In this study, we sought to characterize the rates of early EAC over the past decade when the specific T-stage data became available. The proportion of early, potentially resectable disease was $18.70 \%$ overall, suggesting that approximately one in five patients may be candidates for minimally invasive, organ sparing, endoscopic resection. 


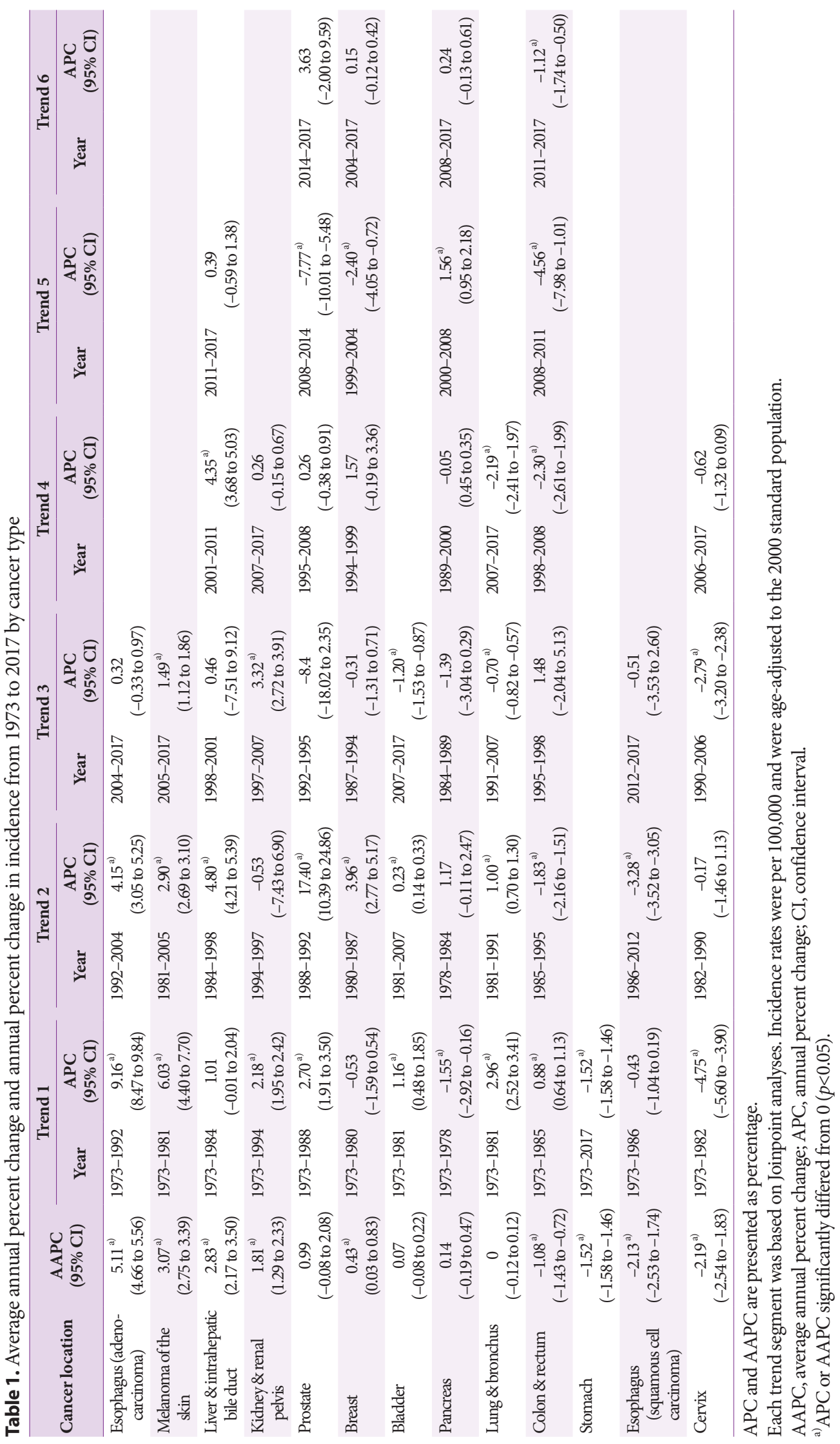




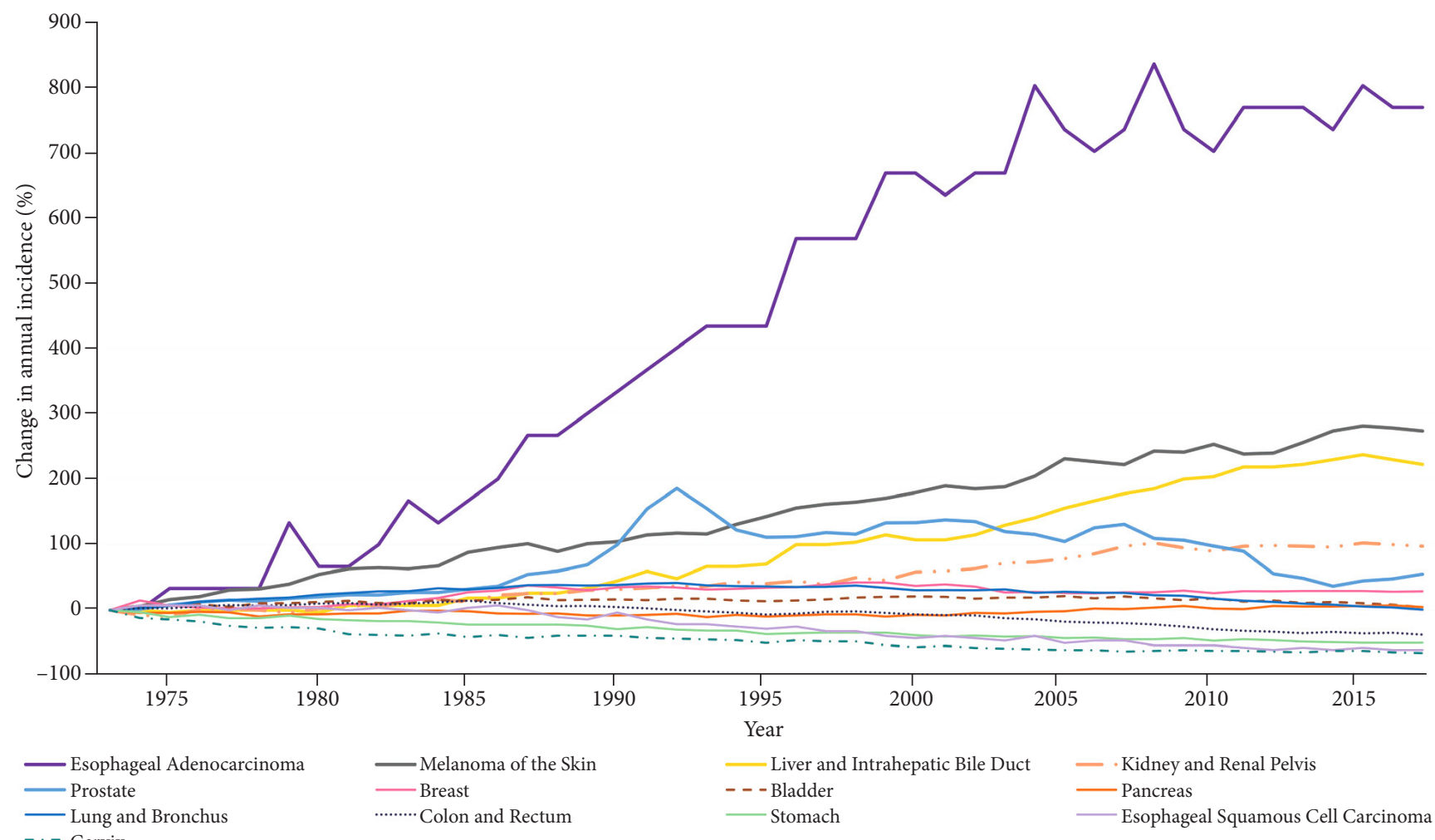

-. - Cervix

Fig. 1. Percent change in annual incidence of major epithelial cancers from 1973-2017 by cancer type relative to the index year. Each epithelial cancer was defined according to the International Classification of Diseases for Oncology (ICO-3)/World Health Organization (WHO) 2008 anatomic locations. The change in the incidence rate for each epithelial malignancy relative to its historic incidence rate in 1973 was represented by the formula: $100 \times[($ rate at a specific year -1973 rate $) / 1973$ rate $]$.

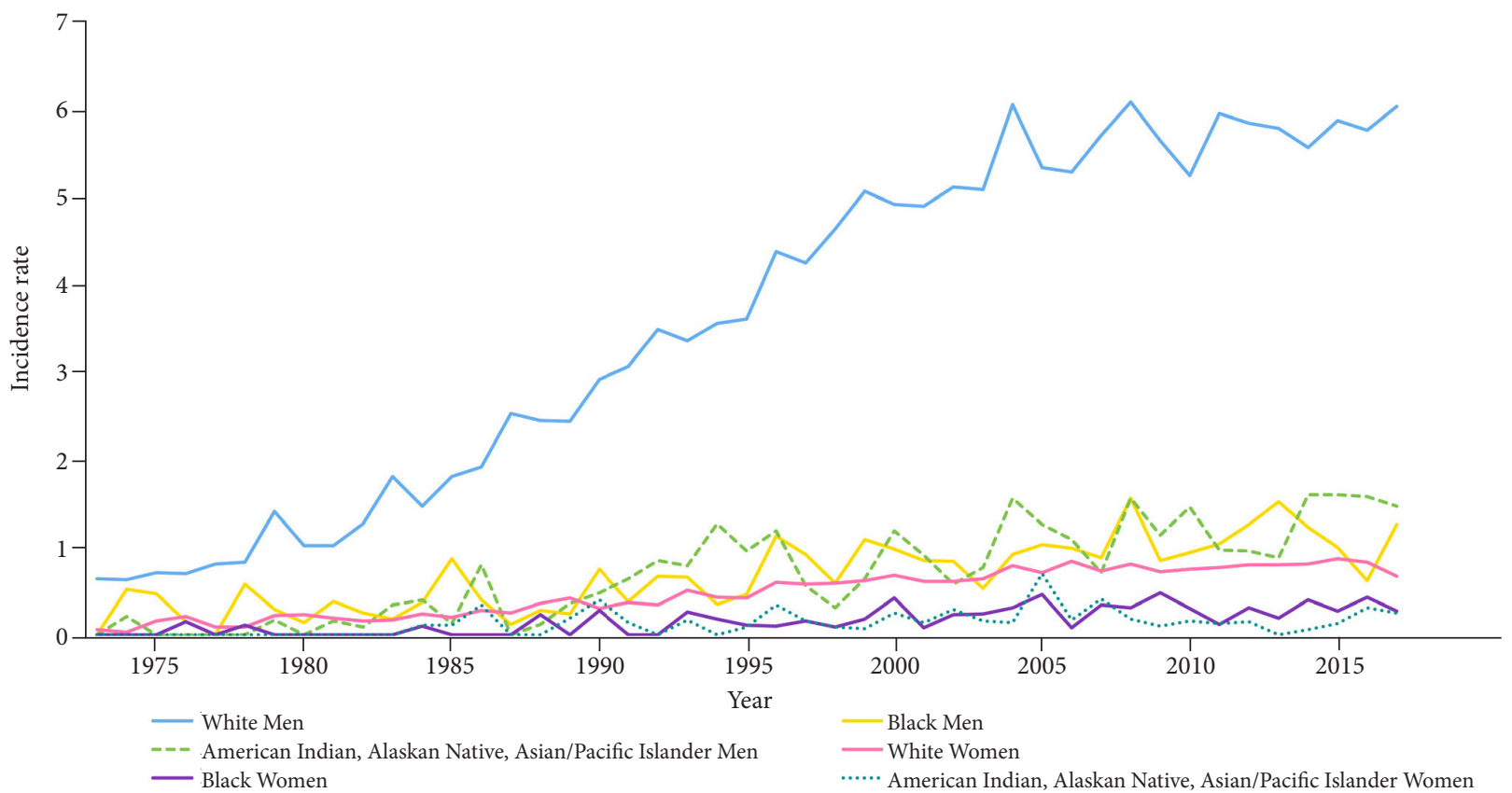

Fig. 2. Incidence rate of esophageal adenocarcinoma from 1973-2017 by race and sex. Incidence rates were per 100,000 individuals and age-adjusted to the 2000 US population. 


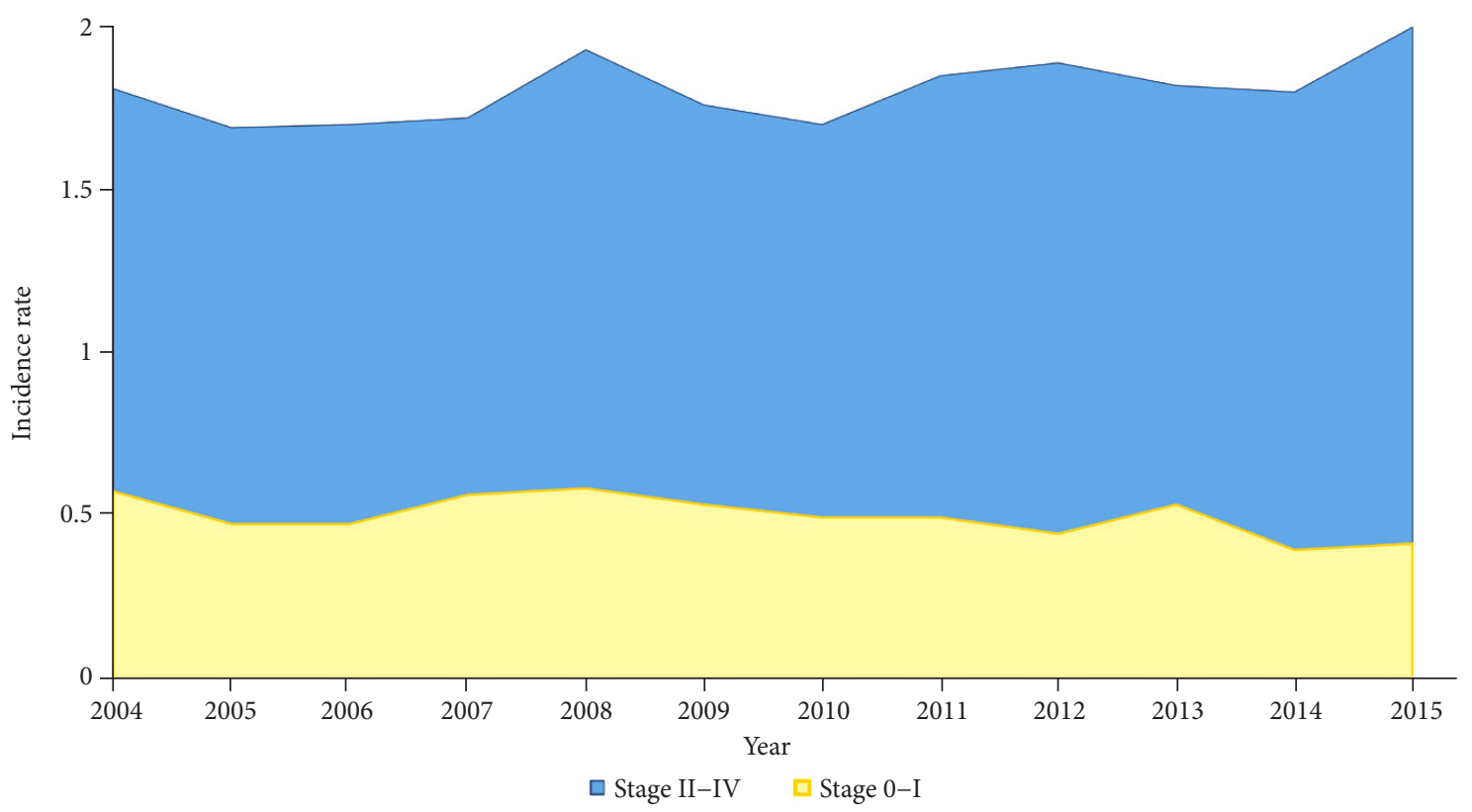

Fig. 3. Incidence rate of early (stage 0 and I) vs stage II-IV esophageal adenocarcinoma from 2004-2015. Staging was based on the American Joint Committee on Cancer Staging, 6th edition. Early stage esophageal adenocarcinoma (stage 0-I) is defined as Tis, T1a, and T1b N0 M0 tumors.

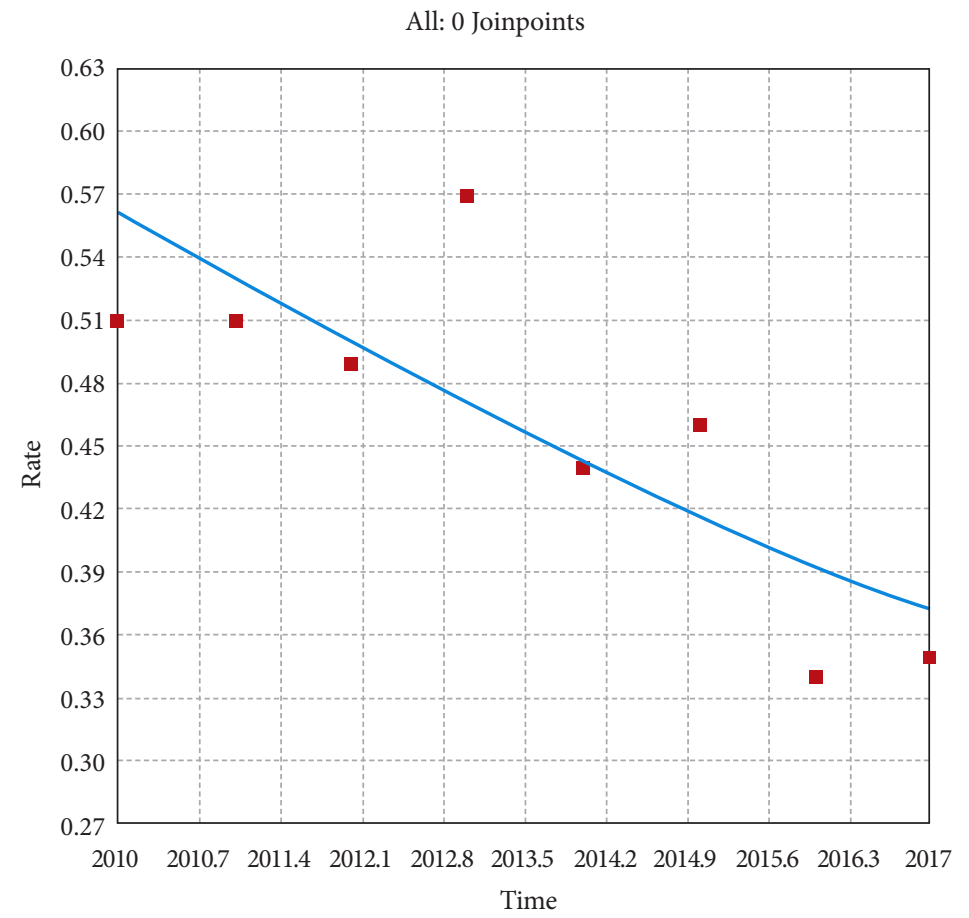

- Observed

- 2010-2017 APC $=-5.78^{\mathrm{a}}$

Fig. 4. Cases of esophageal adenocarcinoma from 2010-2017 by tumor stage. Staging was based on the American Joint Committee on Cancer Staging, 7th edition. ${ }^{\text {a) }}$ Indicates that the annual percent change (APC) is significantly from zero at the alpha $=0.05$ level. Final selected model, 0 Joinpoints.
Concurrent with this increase in disease burden, there has been a proliferation of endoscopic approaches for early stage cancers. Early stage EACs may be addressed with endoscopic mucosal resection followed by radiofrequency ablation, sparing the need for a radical esophagectomy. ${ }^{6}$ Several small prospective studies have reported favorable outcomes for Tis, T1a, and certain T1b tumors in terms of mortality, eradication of cancer, and absence of recurrence. ${ }^{17-23} \mathrm{~A}$ large prospective study of en- 


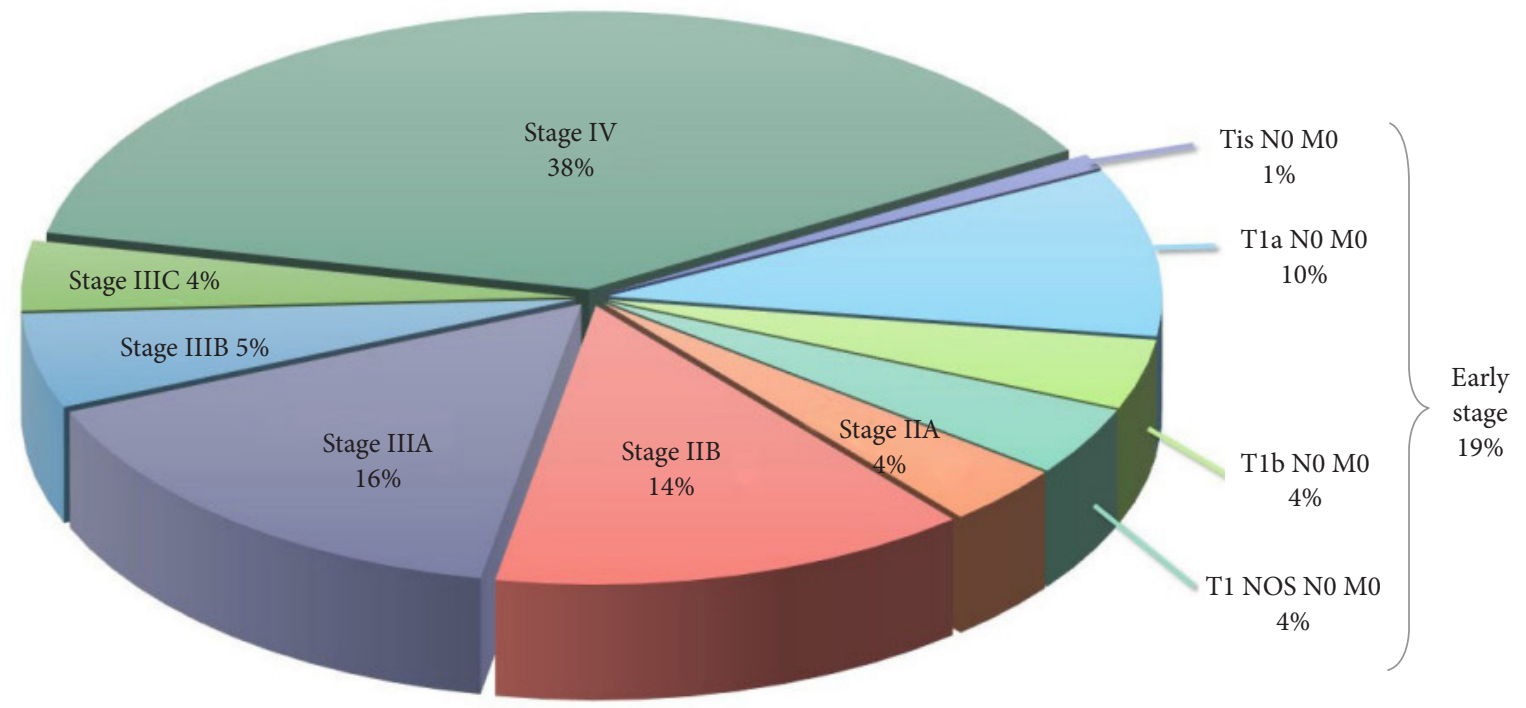

Fig. 5. Annual percent change in early esophageal adenocarcinoma incidence from 2010 to 2017. Staging was based on the American Joint Committee on Cancer Staging, 7th edition. Each trend segment was based on Joinpoint analyses. Incidence rates were per 100,000 and were age-adjusted to the 2000 standard population.

doscopic therapy for T1a EAC in 1,000 patients demonstrated a response rate of $96.3 \%$ over a mean follow-up period of 56.6 months. ${ }^{24}$ For early EAC $\leq 2 \mathrm{~cm}$ in size, the rates of lymph node metastasis are low relative to the mortality associated with esophagectomy. ${ }^{25}$

Good outcomes with endoscopic approaches have led to revisions in the national guidelines, which now favor endoscopic resection for Tis and T1a lesions, followed by radiofrequency ablation. ${ }^{26}$ Our data suggest that approximately $19 \%$ of incident cases of EAC are early and potentially amenable to endoscopic management (Fig. 4). This fraction has been decreasing over the past decade, suggesting that the rising incidence rates are not driven by the early detection of small superficial tumors. Cases detected in this early subset have the best overall survival and are most likely to be associated with a curative outcome. Although improved survival has been reported for patients with localized presentations, there has been relatively little improvement in survival in patients with regional spread or distant metastasis. ${ }^{5,27}$ The median survival rate for patients with distant EAC has remained at 4-6 months since 1970 compared to the more than 3-fold increase in survival with localized EAC at 35 months.

We believe that the increasing rates of EAC in the US population over decades may be driven by the increasing incidence of obesity in the US population. Obesity itself has been linked to tumorigenesis, ${ }^{28,29}$ and in the case of EAC, may also potentiate gastroesophageal reflux disease, ${ }^{30-32}$ the precursor condition to Barrett's esophagus.

Over the final decade of the analysis, there appears to have been a plateau in the overall rate of EAC and a decrease in the rate of early EAC. The rate of increase of EAC appears to have slowed from 2004 to 2017 relative to the steeper rise seen between 1973 and 1997. This may represent a true reduction in the rate of change or may simply reflect variability in the more recent time periods. We postulate that the current plateau in all cases could be due to the long-term effects of proton pump inhibitors, which can help mitigate the inflammation caused by reflux. It is also possible that with widespread proton pump inhibitor use and symptomatic improvement, fewer early stage diseases may be detected, as endoscopy might not be pursued initially.

When it becomes available, an analysis of the incidence data of the upcoming years may clarify the trajectory of EACs. If the plateau in the rate of EAC is persistent over time, several hypotheses could be considered, including the downstream results of efforts directed towards Barrett's screening, surveillance, and eradication. It does not appear that node-negative early T-stage tumors are driving the increasing incidence rates overall, as the rates of early EAC have generally trended with the overall EAC rates over the last decade of analysis.

This study has several limitations. The study was retrospective, and although the data included in this analysis had T-stage 
and nodal status data, details such as lymphovascular invasion were not available in the SEER database. Patients with lymphovascular invasion are not ideal candidates for endoscopic treatment, and a subset of these patients with early stage disease may be re-triaged in clinical settings for surgical management. The data also did not include variables that would have been helpful for speculating on the cause of the increase in EAC rates over time, including whether the diagnoses were established as a part of screening efforts for Barrett's esophagus. Finally, a pathologic review was performed at each individual site and was not centralized.

The rate of EAC has increased more than 7-fold, making it the most rapidly increasing epithelial malignancy in the US. The increasing rates were seen most dramatically in the white male cohort. The incidence of early stage disease also appears to decrease over the past decade. Approximately one in five patients with EAC appears to have early stage disease and may potentially be eligible for organ-sparing, minimally invasive, endoscopic management. While previous studies have examined the overall trend in incidence rates for EAC, this study provides novel information on the fraction of patients with early stage disease who may now be managed with local endoscopic resection and ablation. Further studies are needed to clarify the cause of the disproportionate increase of EAC cases. These data support the proliferation of treatment options and increasing focus on the prevention, detection, and treatment for EAC.

\section{Conflicts of Interest}

The authors have no potential conflicts of interest.

\section{Funding}

This work was supported by a development grant from the Department of Medicine at Emory University School of Medicine. This work was also supported by grant P30CA138292 from the Biostatistics and Bioinformatics Shared Resource of Winship Cancer Institute of Emory University and NIH/NCI. The content is solely the responsibility of the authors and does not necessarily represent the official views of the National Institutes of Health.

\section{Acknowledgments}

The authors wish to acknowledge Sarah Cristofaro and Dr. Ambreen Merchant for their invaluable assistance in study coordination.

\section{Author Contributions}

Conceptualization: AMG, ZC, SF, SK, NFS, BLR, QC, EFW; Data curation: TVPH, ZS; Formal analysis: TVPH, ZS, ZC, FFW; Supervision: FFW; Validation: TVPH, ZS, ZC, FFW; Visualization: TVPH, ZS; Writing-original draft: TVPH, ZS, AMG, FFW; Writingreview \& editing: TVPH, ZS, ZC, VP, SC, SF, SK, NFS, BER, QC, FFW.

\section{ORCID}

Thuy-Van P. Hang

Zachary Spiritos

Anthony M. Gamboa

Zhengjia Chen

Seth Force

Vaishali Patel

Saurabh Chawla

Steven Keilin

Nabil F. Saba

Bassel El-Rayes

Qiang Cai

Field F. Willingham https://orcid.org/0000-0002-2624-7867 https://orcid.org/0000-0001-9076-5477 https://orcid.org/0000-0003-0483-4654 https://orcid.org/0000-0002-6301-4931 https://orcid.org/0000-0003-4879-1246 https://orcid.org/0000-0002-2890-9457 https://orcid.org/0000-0001-6841-4929 https://orcid.org/0000-0003-2310-1210 https://orcid.org/0000-0003-4972-1477 https://orcid.org/0000-0002-2661-5746 https://orcid.org/0000-0002-9931-5410 https://orcid.org/0000-0002-7071-3001

\section{REFERENCES}

1. American Cancer Society. Key statistics for esophageal cancer [Internet]. Kennesaw (GA): American Cancer Society; 2021 [cited 2021 Dec 7]. Available from: https://www.cancer.org/cancer/esophagus-cancer/about/key-statistics.html.

2. Lagergren J, Lagergren P. Recent developments in esophageal adenocarcinoma. CA Cancer J Clin 2013;63:232-248.

3. Pohl H, Welch HG. The role of overdiagnosis and reclassification in the marked increase of esophageal adenocarcinoma incidence. J Natl Cancer Inst 2005;97:142-146.

4. Hur C, Miller M, Kong CY, et al. Trends in esophageal adenocarcinoma incidence and mortality. Cancer 2013;119:1149-1158.

5. Dubecz A, Gall I, Solymosi N, et al. Temporal trends in long-term survival and cure rates in esophageal cancer: a SEER database analysis. J Thorac Oncol 2012;7:443-447.

6. Barnes JA, Willingham FF. Endoscopic management of early esophageal cancer. J Clin Gastroenterol 2015;49:638-646.

7. National Institutes of Health. SEER ${ }^{\star}$ Stat software (version 8.3.9.2) [Internet]. Bethesda (MD): National Institutes of Health; 2021 [cited 2021 Dec 7]. Available from: https://seer.cancer.gov/seerstat/index. html.

8. Greene FL, Balch CM, Fleming ID, et al. AJCC cancer staging handbook: TNM classification of malignant tumors. 6th ed. New York 
(NY): Springer; 2002.

9. Edge S, Byrd DR, Compton CC, et al. AJCC cancer staging handbook: from the AJCC cancer staging manual. 7th ed. New York (NY): Springer; 2010.

10. National Institutes of Health. Joinpoint regression program [Internet]. Bethesda (MD): National Institutes of Health [cited 2021 Dec 7]. Available from: https://surveillance.cancer.gov/joinpoint/.

11. Murphy CC, Sandler RS, Sanoff HK, et al. Decrease in incidence of colorectal cancer among individuals 50 years or older after recommendations for population-based screening. Clin Gastroenterol Hepatol 2017;15:903-909.e6.

12. Govindan R, Page N, Morgensztern D, et al. Changing epidemiology of small-cell lung cancer in the United States over the last 30 years: analysis of the surveillance, epidemiologic, and end results database. J Clin Oncol 2006;24:4539-4544.

13. Banks E, Canfell K. Recent declines in breast cancer incidence: mounting evidence that reduced use of menopausal hormones is largely responsible. Breast Cancer Res 2010;12:103.

14. He H, Chen N, Hou Y, et al. Trends in the incidence and survival of patients with esophageal cancer: a SEER database analysis. Thorac Cancer 2020;11:1121-1128.

15. Haiyu Z, Xiaofeng P, Xiangqiong M, et al. Incidence and survival changes in patients with esophageal adenocarcinoma during 19842013. Biomed Res Int 2019;2019:7431850.

16. Then EO, Lopez M, Saleem S, et al. Esophageal cancer: an updated surveillance epidemiology and end results database analysis. World J Oncol 2020;11:55-64.

17. Pech O, Behrens A, May A, et al. Long-term results and risk factor analysis for recurrence after curative endoscopic therapy in 349 patients with high-grade intraepithelial neoplasia and mucosal adenocarcinoma in Barrett's oesophagus. Gut 2008;57:1200-1206.

18. Peters FP, Kara MA, Rosmolen WD, et al. Endoscopic treatment of high-grade dysplasia and early stage cancer in Barrett's esophagus. Gastrointest Endosc 2005;61:506-514.

19. Larghi A, Lightdale CJ, Ross AS, et al. Long-term follow-up of complete Barrett's eradication endoscopic mucosal resection (CBE-EMR) for the treatment of high grade dysplasia and intramucosal carcinoma. Endoscopy 2007;39:1086-1091

20. Ell C, May A, Pech O, et al. Curative endoscopic resection of early esophageal adenocarcinomas (Barrett's cancer). Gastrointest Endosc 2007;65:3-10

21. May A, Gossner L, Pech O, et al. Intraepithelial high-grade neoplasia and early adenocarcinoma in short-segment Barrett's esophagus (SSBE): curative treatment using local endoscopic treatment techniques. Endoscopy 2002;34:604-610.

22. Seewald S, Akaraviputh T, Seitz U, et al. Circumferential EMR and complete removal of Barrett's epithelium: a new approach to management of Barrett's esophagus containing high-grade intraepithelial neoplasia and intramucosal carcinoma. Gastrointest Endosc 2003;57:854-859.

23. Manner H, Pech O, Heldmann Y, et al. Efficacy, safety, and long-term results of endoscopic treatment for early stage adenocarcinoma of the esophagus with low-risk sml invasion. Clin Gastroenterol Hepatol 2013;11:630-635.

24. Pech O, May A, Manner H, et al. Long-term efficacy and safety of endoscopic resection for patients with mucosal adenocarcinoma of the esophagus. Gastroenterology 2014;146:652-660.e1.

25. Gamboa AM, Kim S, Force SD, et al. Treatment allocation in patients with early-stage esophageal adenocarcinoma: prevalence and predictors of lymph node involvement. Cancer 2016;122:2150-2157.

26. Ajani JA, D’Amico TA, Almhanna K, et al. Esophageal and esophagogastric junction cancers, version 1.2015. J Natl Compr Canc Netw 2015;13:194-227.

27. Siegel RL, Miller KD, Jemal A. Cancer statistics, 2020. CA Cancer J Clin 2020;70:7-30.

28. De Pergola G, Silvestris F. Obesity as a major risk factor for cancer. J Obes 2013;2013:291546.

29. Nieman KM, Romero IL, Van Houten B, et al. Adipose tissue and adipocytes support tumorigenesis and metastasis. Biochim Biophys Acta 2013;1831:1533-1541.

30. Chang P, Friedenberg F. Obesity and GERD. Gastroenterol Clin North Am 2014;43:161-173.

31. Ness-Jensen E, Hveem K, El-Serag H, et al. Lifestyle intervention in gastroesophageal reflux disease. Clin Gastroenterol Hepatol 2016;14:175-182.e1-e3.

32. Schlottmann F, Dreifuss NH, Patti MG. Obesity and esophageal cancer: GERD, Barrett's esophagus, and molecular carcinogenic pathways. Expert Rev Gastroenterol Hepatol 2020;14:425-433. 